\title{
The Stability Threshold and Two Facets of Polarization*
}

\author{
Ori Haimanko $\quad$ Michel Le Breton ${ }^{\ddagger} \quad$ Shlomo Weber ${ }^{\S}$ \\ March 2006
}

\begin{abstract}
In this paper we introduce the stability threshold that quantifies the minimal returns to size sufficient to prevent credible secession threats by regions of the country. Severity of internal tension has been linked to degree of polarization of citizens' preferences and characteristics. We show that the increasing degree of polarization does not, in general, raise the stability threshold, even though this hypothesis holds in some asymptotic sense. We also examine the question of the number of smaller countries to be created if the unity of the large country is not sustainable, and investigate the link between this number and the degree of the country polarization.
\end{abstract}

Key Words: Polarization, Secession, Stability threshold, Clusters.

JEL Classification Numbers: H20, D70, D73.

*We wish to thank an anonymous referee for useful comments.

${ }^{\dagger}$ Ben-Gurion University, Israel.

¥Université de Toulouse I, GREMAQ and IDEI, Toulouse, France.

§outhern Methodist University, USA, CORE, Université Catholique de Louvain, Belgium, and CEPR. 


\section{Introduction}

Internal conflicts over government policies often threaten the stability of a country. Indeed, dissatisfied regions of the country may attempt to secede, if the economies of scale brought by unity are overweighed by the benefits of forming a separate entity (in which the level of internal confrontation is reduced, or eliminated altogether). In many cases, these conflicts are created by the lack of uniformity in citizens' preferences over the range of government policy choices and/or distinctions across ethnic, religious, historical or linguistic lines. Thus, stability of the country is linked to the distribution of its citizens' preferred policies or characteristics.

How stability of the country should be measured? We suggest to measure it by means of stability threshold. This index quantifies the minimal returns to size that are sufficient to prevent credible secession threats. An alternative interpretation of the stability threshold is that of the minimal burden that can be imposed on the country (and all regions, provided they decide to secede) which still guarantees its unity.

Our notion of stability, that requires "secession-proofness" of the country in the face of internal conflicts, effectively ties stability with the precise form of the society's conflictinducing diversity, represented by the distribution of citizens' preferences and characteristics. Thus, the stability threshold can also serve as a measure of severity of internal conflicts. ${ }^{1}$ This last concept has already been quantified by Esteban and Ray (1999), as the total equilibrium expenditure by individuals on internal struggle (aimed at promoting their own most preferred alternative). But we think that it is also natural to take a complementary view and assess the severity of a conflict by the strength of secession threats that this conflict generates. This strength is faithfully represented by the stability threshold, or the size of overall resources that can prevent or at least mitigate secession sentiment.

\footnotetext{
${ }^{1}$ For the literature on "greed-based" conflicts motivated by competition over resources see Grossman (1991), Gershenson and Grossman (1999), Caselli and Coleman (2002).
} 
Severity of internal tension has been linked in the literature to polarization of the distribution of citizens' preferences and characteristics. The common belief (Esteban and Ray (1994, 1999), Duclos et al. (2004)) is that raising the degree of polarization increases the probability of internal conflicts, which should thus make secession threats by the country's dissatisfied regions more severe. It therefore seems proper to check this hypothesis, by enquiring into the relation between the degree of country's polarization and its stability threshold (which as was said is also a measure of severity of internal conflicts).

Our main finding is that, somewhat counter-intuitively, the relation between polarization and the stability threshold is ambiguous. Recall (Esteban and Ray (1994)), that the concept of polarization is based on the existence of several population clusters with relative homogeneity of preferences within clusters and substantial heterogeneity across clusters. The overall measure of polarization is then determined by the following two factors. The first is the level of heterogeneity inside each cluster (for a given number of clusters), that represents the degree of polarization and conflict between existing population groups: the less heterogeneous each cluster is, the more polarized is the society at large. The second is reflected by the number of clusters in the society, when a smaller (but greater than one) number of clusters represents a higher degree of confrontation (and polarization) in the society. Dependence of the polarization index on the first factor will be called fixed-clusters polarization effect (FCPE), and on the second factor - variable-clusters polarization effect (VCPE). Our basic conclusions are as follows:

- The stability threshold of a country is positively correlated with the FCPE.

- The link between the stability threshold and VCPE is ambiguous.

- The impact of VCPE is sufficiently strong so that the combined effect of FCPE and VCPE is ambiguous as well.

- However, there is positive correlation between the stability threshold and the polarization when the polarization is low, which happens when the number of clusters is sufficiently 
large and each cluster is sufficiently heterogeneous.

The somewhat unexpected behavior of the stability threshold with respect to VCPE is due to the following reason. Existence of a "centrally-located" cluster (the one where the preferences fall in, or close to, the center of the political map) can make secessions more difficult compared to the situation when the center is "vacant". This is because the central cluster benefits the most from being in a united country (since the chosen policy would typically be geared towards the "median" citizen). It may therefore be costly to persuade this cluster to join a seceding region (if it is too small to profit from secession by itself), or the citizens of that region may actually favor unity because then they can demand compensation from the politically-satisfied central cluster. Thus, the stability threshold may increase when the country's population undergoes a division into more clusters (although located closer to each other) and the center becomes vacant, despite a decrease in polarization. This, as was said, cannot happen in the case of FCPE, and also not when the population preferences are distributed very uniformly across their range.

The behavior of the Esteban and Ray (1999) measure of societal conflict also exhibits a certain lack of monotonicity with respect to changes in polarization of a multi-cluster society. However, their focus is, to a certain degree, on "contests" (where individuals have utility only from their most preferred alternative). In their model, the only option at an individual's disposal consists of lobbying for the acceptance of his ideal policy, without the possibility of a secession. Our analysis captures a different aspect of a conflict - the strength of secession threats that it generates - and shows a certain divergence between it and the polarization index of the society, as we explained above. However, in spite of the differences between the framework of Esteban and Ray (1999) and our set-up, both approaches converge in their analysis of the fixed-cluster effect. Indeed, for a given number of clusters (three in Esteban and Ray (1999)) both indices of conflict severity are positively correlated with the degree of dissimilarity between the clusters, and, thus, represent reasonable indicators for the intensity 
the conflict at hand.

In the second part of the paper we examine the situation where the stability threshold has not been achieved and the country faces a break-up. We then examine the stable number of countries, i.e., the number of independent entities into which the given united country should be broken in order to eliminate credible threats of secession. ${ }^{2}$ We find that the stable number of countries also behaves non-monotonically with respect to polarization indices. However, monotonicity does appear when the stable number is large, and the stable number decreases when polarization rises.

Like Esteban and Ray's paper, this manuscript aims to offer a theoretical contribution to the comparative statics of social equilibrium : how the equilibrium of a particular set of social institutions responds to modifications of one or several societal parameters describing the community under scrutiny. It sheds light on the difficulties attached to the question and show that "natural" monotonicity properties should be considered with caution. These questions have been also investigated from an empirical perspective. In a model similar to the one considered in this paper, but with two policy dimensions instead of one (both the type and the quantity of the public good have to be selected), Alesina, Baqir and Easterly (1999) show that the quantity of public good in the social equilibrium resulting from sequential majority voting is inversely related to the median distance to the median that they consider as being an indicator of polarization of preferences. They test this implication with three data sets (U.S. cities, U.S. metropolitan areas and U.S. urban counties) and bring evidence that ethnic conflict and fragmentation (there, they use the ethnic fractionalization index) is an important determinant of local public finance. Alesina, Baqir and Hoxby (2004) investigates the relationship between the number of jurisdictions

\footnotetext{
${ }^{2}$ See Alesina and Spolaore (1997) in the case of the uniform distribution of citizens' characteristics.
} 
and various measures of social heterogeneity in a model which builds on Alesina and Spolaore. They test the model using American school districts, school attendance areas, municipalities and special districts and find strongevidence of the impact of social and income heterogeneity but little evidence for ethnic or religious heterogeneity.

The paper is organized as follows. Section 2 contains the formal model of a country with heterogeneous citizens and the definition of stability threshold. In Section 3 we discuss the notion of polarization. Our results on the link between stability and polarization are presented in Section 4, whereas Section 5 studies stability in the multi-country framework and its relation to polarization. The proofs are relegated to the Appendix.

\section{Model}

We consider a country $\mathcal{W}$ with a population of total mass 1 , whose citizens have preferences over the unidimensional policy space given by the interval $I=[0,1]$. Citizens have symmetric single-peaked preferences over the set $I$, and we identify each citizen with her ideal point (and thus $\mathcal{W} \equiv I$ ). The distribution of all ideal points (and, thus, of all citizens' preferences) is given by a cumulative distribution function $F$ with density $f$, defined over $I$.

The country $\mathcal{W}$ chooses a policy in the policy space $I$. In this paper, as in Alesina and Spolaore (1997) and Le Breton and Weber (2003), we adopt a spatial interpretation of the model by identifying a policy with the physical location of the government, so we do not distinguish between geographical and preference dimensions. The country $\mathcal{W}$ has to cover the cost of provision of public good, or government cost, $c$. We assume that the government costs are the same for all regions, and if a region secedes from $\mathcal{W}$, it will have to cover the same cost $c$. For simplicity, we restrict our analysis of possible secessions regions that consist of the union of a finite number of closed intervals in $\mathcal{W}$.

Suppose now that an individual $t$ belongs to the set $S$, which could be either the unified 
country $(S=\mathcal{W})$ or a seceding region $(S \subseteq \mathcal{W})$, and whose government chooses a location $p \in I$. Then the disutility or "transportation" cost $d(t, p)$, incurred by individual $t$ from the choice of $p$, is determined by the distance between $t$ and the government location $p$ and we shall assume that:

$$
d(t, p)=|t-p|
$$

Now denote

$$
D(S, p)=\int_{S} d(t, p) f(t) d t
$$

Then the value

$$
D(S)=\min _{p \in I} \int_{S} d(t, p) f(t) d t
$$

represents the minimal aggregate transportation cost incurred by the citizens of $S .{ }^{3}$

Under the linearity assumption, the aggregate transportation cost for every set $S$ is minimized when the government location chooses its location at the ideal point of its "median citizen", $m(S)$, that satisfies $\int_{\{t \in S \mid t \leq m(S)\}} f(t) d t=\int_{\{t \in S \mid t \geq m(S)\}} f(t) d t$. Note that if $S$ is an interval and $f$ is positive on $S$, then its median citizen is uniquely defined. However, if $S$ consists of several disjoint intervals, the median of $S$ is not necessarily unique.

We now introduce the notion of $S$-cost allocation that determines the monetary contribution of each individual $t$ towards the cost of government $c$.

Definition 2.1: A bounded measurable function $x$ defined on the set $S \subseteq \mathcal{W}$ is called an $S$-cost allocation if it satisfies the budget constraint:

$$
\int_{S} x(t) f(t) d t=c .
$$

When the government location of $S$ is at $p$, the total disutility of citizen $t \in S$ under $S$-cost allocation $x$ is:

$$
d(t, p)+x(t)
$$

\footnotetext{
${ }^{3}$ There always exists an optimal location of the government (see the next paragraph) and, therefore, the cost function is well defined.
} 
We allow for lump sum transfers and do not restrict the mechanism of sharing costs in any way. Thus every region $S$ that contemplates secession, could take into account only its total cost of being a separate country, given by the sum of government and transportation costs, in estimating its future gains:

$$
c+D(S)
$$

If region $S$ (which is, to recall, a union of a finite number of subintervals of $I$ ) can make its members better off than under the central government, then $S$ is said to be prone to secession:

Definition 2.2: Consider a pair $(p, x)$, where $p$ is the location of national government and $x$ is a $\mathcal{W}$-cost allocation. We say that region $S$ is prone to secession (given $(p, x)$ ) if

$$
\int_{S}(d(t, p)+x(t)) f(t) d t>D(S)+c .
$$

If no region is prone to secession, then the pair $(p, x)$ is called secession-proof. The country is called stable if there exists a secession-proof pair $(p, x)$.

We now introduce stability threshold or unity index that quantifies the minimal returns to size that are sufficient to prevent credible secession threats. As we mentioned in the introduction, this threshold can be viewed as the minimal burden on the country which still guarantees its unity. It is quite easy to observe that the notions of stability and secessionproofness are closely linked to the cost of public good. Indeed, a high cost of public good may facilitate regional cooperation and mitigate a threat to instability posed by regions. On the other hand, a low cost of public goods could reduce incentives for economic unity and raise the intensity of secession threats. Formally,

Proposition 2.3: For a given distribution of ideal points $F$, there is a cut-off value of government $\operatorname{costs} c^{s t}(F)$ such that the country is stable (according to Definition 2.1) if and only if $c \geq c^{s t}(F)$. 
The value $c^{s t}(F)$ is called the stability threshold of $F$. Thus, for any given government cost $c \geq c^{s t}(F)$, the country is stable: there exists a government location $p$ and a $\mathcal{W}$-cost allocation $x$ such that no region is prone to secession given $(p, x)$. But, if $c<c^{s t}(F)$, the country is unstable: there exists no secession-proof pair $(p, x)$.

The natural question we address in this paper is the investigation of the link between the stability threshold a degree of the country's polarization. In order to do so, in the next section we proceed with examination of polarization index.

\section{Polarization Index}

Indices of polarization, introduced in Esteban and Ray (1994), Duclos, et al. (2004) Tsui and Wang (2000), are based on the notions of identification within one's own group and alienation towards the others. For a continuous cumulative distribution function $F$ on $[0,1]$, Duclos et al. (2004) have derived the following polarization index $\gamma_{\alpha}(F)$ :

$$
\gamma_{\alpha}(F)=\int_{0}^{1} \int_{0}^{1}|x-y| f(x)^{1+\alpha} f(y) d x d y,
$$

where $f$ is the density function of $F$, and the parameter $\alpha$ satisfies $0.25 \leq \alpha \leq 1$. If $F$ is a discrete distribution supported on the set $\left\{x_{0}, \ldots, x_{n}\right\}$, and $p_{i}$ is the probability of $x_{i}$, the index (derived by Esteban and Ray (1994)) is given by

$$
\gamma_{\alpha}(F)=\sum_{i=0}^{n} \sum_{j=0}^{n} p_{i}^{1+\alpha} p_{j}\left|x_{i}-x_{j}\right|,
$$

where the parameter $\alpha$ belongs to the interval $\left[0, \alpha^{*}\right]$, where $\alpha^{*} \approx 1.6$. To cover both infinite and finite cases, we assume throughout the rest of the paper that $0.25 \leq \alpha \leq 1$, so that both (1) and (2) hold.

As alluded to in the introduction, our analysis of conflicts will be performed under the assumption that citizens' ideal points form several disjoint clusters (that represent geographical regions or groups with similar political views). This will highlight the following 
two attributes of conflict situations (in addition to the existence of clusters). The first is heterogeneity of preferences within clusters, which represents conflicts within each region or group. The second is reflected by the number of distinct groups within the society, when a smaller (but greater than one) number of clusters represents a higher degree of confrontation. In order to focus solely on these two factors and eliminate other effects, we shall consider a family of step distribution functions with the support over a finite number of equal intervals (clusters). We shall also assume complete uniformity of the distribution of citizens' ideal points within each cluster. Thus, all distributions in our class $\mathcal{F}$ will be characterized by two parameters, the number of clusters, $n$ and their length, $a$.

Formally, let an integer $n \geq 2$ and the parameter $a \in\left(0, \frac{1}{n}\right]$ be given. Consider a function $f_{n, a}$ on the unit interval $[0,1]$ :

$$
f_{n, a}(t)= \begin{cases}\frac{1}{n a} & \text { if } t \in\left[j \frac{1-a}{n-1}, j \frac{1-a}{n-1}+a\right] \text { for } j=0,1, \ldots, n-1 \\ 0 & \text { otherwise. }\end{cases}
$$

That is, $f_{n, a}$ is the density function of the distribution which is supported and uniform on the $n$ intervals of length $a$, removed from each other by the same distance. Denote the corresponding distribution by $F_{n, a}$. We also introduce $\left\{F_{n, 0}\right\}$ for $n \geq 2$, which is a discrete limiting distribution of $\left\{F_{n, a}\right\}$ for $a \in\left(0, \frac{1}{n}\right]$. That is, $F_{n, 0}$ is supported, and is uniform, on the finite set that consists of $n$ equidistant points $\left\{0, \frac{1}{n-1}, \frac{2}{n-1}, \ldots, \frac{n-1}{n-1}=1\right\}$.

Now, as in Duclos et al. (2004), let $0.25 \leq \alpha \leq 1$, and denote $\gamma_{\alpha}(n, a) \equiv \gamma_{\alpha}\left(F_{n, a}\right)$. We have the following expression for the polarization index:

\section{Proposition 3.1:}

$$
\gamma_{\alpha}(n, a)= \begin{cases}\left(\frac{1}{n a}\right)^{\alpha} \frac{n+1-n a}{3 n}, & \text { if } a>0 \\ \left(\frac{1}{n}\right)^{\alpha} \frac{n+1}{3 n}, & \text { if } a=0\end{cases}
$$

Obviously, the distribution $F_{n, a}$ becomes less polarized when $a$ or $n$ increase:

Corollary 3.2: The polarization index $\gamma_{\alpha}(n, a)$ declines in each of its two variables. 
According to our interpretation in the introduction, the dependence of $\gamma_{\alpha}(n, a)$ on $a$ describes the fixed-clusters polarization effect (FCPE), while its dependence on $n$ reflects the variable-clusters polarization effect ( $V C P E)$. Thus, both effects reduce the polarization index.

It is worth pointing out that the index $\gamma_{\alpha}$ exhibits discontinuity in the transition from continuous distributions $F_{n, a}$ for $a>0$ to $F_{n, 0}: \lim _{\alpha \searrow 0} \gamma_{\alpha}(n, a)=\infty$. The reason is that according to this index discrete distributions are infinitely more polarized than continuous ones (due to the presence of infinitely dense clusters in former). The index still allows comparisons of discrete distributions $\left\{F_{n, 0}\right\}$, via $(3)$, but they belong to a different (higher) league of polarization when it comes to comparing them with continuous distributions $\left\{F_{n, a}\right\}$. The index should not therefore be used for comparisons across these two subfamilies of distributions, but only for comparisons inside each subfamily.

\section{The Linkage between the Stability Threshold and Polarization}

In this section we study how the stability threshold reacts to changes in polarization. First, we explicitly calculate the stability threshold for the distributions in our class. For every function $F_{n, a} \in \mathcal{F}$ we shall use a notation $c^{s t}(n, a)$ instead of $c^{s t}\left(F_{n, a}\right)$.

Proposition 4.1: For $n \geq 2, a \in\left[0, \frac{1}{n}\right]$, the stability threshold $c^{s t}(n, a)$ is given by:

$$
c^{s t}(n, a)=\frac{1}{8}\left(1+(1-a n) \frac{1+\frac{4}{n}\left(\left[\frac{n+2}{4}\right]-\left[\frac{n+1}{4}\right]\right)}{2\left[\frac{n-1}{2}\right]+1}\right),
$$

where $[x]$ stands for the integer part of $x$, i.e., for the largest integer that does not exceed $x$.

We now turn to our conclusions:

Proposition 4.2: (i) The stability threshold is positively correlated with FCPE. That is, the increase in $a$ for fixed $n$ reduces both the polarization index and the stability 
threshold.

(ii) The link between the stability threshold and VCPE is ambiguous. That is, while an increase in $n$ reduces the polarization index $\gamma_{\alpha}(n, a)$, it does not necessarily reduce, or increase, the stability threshold $c^{s t}(n, a)$ for fixed $a$.

(iii) The VCPE is strong enough to make the combined effect of FCPE and VCPE on the stability threshold ambiguous as well. That is, even if both $n$ and $a$ increase, thereby reducing the polarization index $\gamma_{\alpha}(n, a)$, this does not necessarily reduce, or increase, the stability threshold $c^{s t}(n, a)$.

Thus, in general, the relationship between polarization and stability is not monotone.

According to Proposition 4.2, the stability threshold of $F_{n, a}$ decreases with the increase of $a$ (and the implied fall in the distribution's polarization) for fixed $n$, but, in general, is not monotone in $\gamma(n, a)$ for a given $a$. To illustrate this point, consider the finite case $a=0$ and denote by $S(n, t)$ the set of citizens that for given $n$ are located at $t$. As it is shown in the proof of Proposition 4.2, the first deviation from monotone decline of $c^{s t}(n, 0)$ in $n$ occurs when $n=6$. Indeed, $c^{s t}(6,0)=\frac{1}{6}>c^{s t}(5,0)=\frac{3}{20}$. The reason for this spike, alluded to in the introduction, can be explained as follows. When $n=5$, the "central cluster" $S\left(5, \frac{1}{2}\right)$ (which does not exist when $n=6$ ) makes secessions difficult. Indeed, in the united country scenario the optimally chosen government location is also at the center ${ }^{4}$. The existence of a relatively big central cluster (which incurs zero transportation cost) has a mitigating effect on the aggregate transportation cost burden. And, if we consider the sets $S(5,0) \cup S\left(5, \frac{1}{4}\right)$ or $S\left(5, \frac{3}{4}\right) \cup S(5,1),{ }^{5}$ none has a "central block" with zero transportation cost. This means that these regions would incur quite high transportation costs in the case of secession. However, the situation changes drastically when $n=6$. In this scenario, there is no central cluster

\footnotetext{
${ }^{4}$ It is easily to verify that, under the linearity assumption, in a secession-proof pair $(p, x)$ the government location $p$ must be the ideal point of the "median citizen" $m(I)=\frac{1}{2}$.

${ }^{5}$ Our proofs indicate that only these subintervals and their complements play a role in determination of $\mathcal{W}$ 's stability - see Lemma A.2 in the Appendix.
} 
in $I$ to mitigate aggregate transportation cost; on the other hand, each of the "secessionrelevant" regions $S(6,0) \cup S\left(6, \frac{1}{5}\right) \cup S\left(6, \frac{2}{5}\right)$ or $S\left(6, \frac{3}{5}\right) \cup S\left(6, \frac{4}{5}\right) \cup S(6,1)$ have central clusters which help to reduce transportation costs in the case of secession. This makes secession more likely and the country less stable for $F_{6,0}$, compared to the more polarized distribution $F_{5,0}$. (This argument can only be made for the switch from $n=4 m+1$ to $4 m+2$ for a positive integer $m$. Indeed, we only observe upward jumps in $c^{s t}(n, 0)$ at $n=4 m+2$ as the proof of Proposition 4.2 will show. To see why the argument cannot be extended, consider for instance the case of $n=3,4$. When $n=3$, aggregate transportation costs are mitigated by the central cluster, $S\left(3, \frac{1}{2}\right)$, but the extreme clusters $S(3,0)$ and $S(3,1)$ would now incur no transportation costs in case of secession, and so are relatively less deterred from seceding compared to the case of $n=5$. And when $n=4$, there is no central cluster to mitigate the transportation cost, but $S(4,0) \cup S\left(4, \frac{1}{3}\right)$ or $S\left(4, \frac{2}{3}\right) \cup S(4,1)$ do not have central clusters either, which reduces their incentives to secede compared to the case of $n=6$. By Proposition 4.1, here $c^{s t}(4,0)=\frac{1}{6}=c^{s t}(3,0)$, and so indeed the less polarized country is not less stable.)

It is worthwhile to note that, for a positive fixed $a$, the decline of $c^{s t}(n, a)$ in $n$ is restored if the value of $n$ is large enough (and thus polarization is low):

Proposition 4.3: For every $0<a<1$, there exists a value $n(a)$ such that $c^{s t}\left(n_{1}, a\right) \leq$ $c^{s t}\left(n_{2}, a\right)$ whenever $n_{1}>n_{2}>n(a)$ and $n_{1} a \leq 1$.

\section{The Stable Number of Countries and Polarization Indices}

When the government cost is low, $\mathcal{W}$ is no longer stable (Proposition 2.3) and could be broken up into smaller entities. The question we analyze in this section is what is the number of smaller countries that could guarantee the stability of partition of $\mathcal{W}$ :

Definition 5.1: Consider a partition $\left(S_{1}, \ldots, S_{m}\right)$ of $\mathcal{W}$ into $m$ countries, an $m$-tuple of pairs 
$\left(\left(p_{1}, x_{1}\right), \ldots,\left(p_{m}, x_{m}\right)\right)$, where $p_{i}$ is the government location in $S_{i}$ and $x_{i}$ is an $S_{i}$-cost allocation. We say that region $S$ is prone to secession (given $\left.\left(\left(p_{1}, x_{1}\right), \ldots,\left(p_{m}, x_{m}\right)\right)\right)$ if

$$
\sum_{i=1}^{m} \int_{S \cap S_{i}}\left(d\left(t, p_{i}\right)+x_{i}(t)\right) f(t) d t>D(S)+c .
$$

If no region is prone to secession, then the $m$-tuple $\left(\left(p_{1}, x_{1}\right), \ldots,\left(p_{m}, x_{m}\right)\right)$ is called secession-proof. The partition $\left(S_{1}, \ldots, S_{m}\right)$ is called stable if there exists a secessionproof $m$-tuple $\left(\left(p_{1}, x_{1}\right), \ldots,\left(p_{m}, x_{m}\right)\right)$.

Proposition 5.2 below follows from the main result in Haimanko et al. (2004):

Proposition 5.2: For a given distribution of ideal points $F \in \mathcal{F}$ and the government cost $c>0$, there exists a stable partition $\left(S_{1}, \ldots, S_{n}\right)$ of $\mathcal{W}$.

In particular, when $c \geq c^{s t}(n, a)$, the trivial partition of $\mathcal{W}$ (consisting of $\mathcal{W}$ itself) is stable.

Denote by $\bar{K}(c, n, a)$ the maximal number of countries in a stable partition of $I$ (when the distribution of ideal points is $f_{n, a}$ and the government cost is $\left.c\right)$, and by $\underline{K}(c, n, a)-$ the minimal number of countries. For simplicity, we will focus attention on $K(c, n, a)=$ $\underline{K}(c, n, a)$; all our observations apply to $\bar{K}(c, n, a)$ just as well. We shall call $K(c, n, a)$ the stable number of countries. It is natural to ask how it is affected by the change in $\gamma_{\alpha}(n, a)$, the polarization degree of $f_{n, a}$.

First, it turns out that $K$ does not, in general, behave monotonically in the polarization degree. Indeed, pick $c_{0} \in\left(\frac{3}{20}, \frac{1}{6}\right)$. Then, since $c^{s t}(4,0)=c^{s t}(6,0)=\frac{1}{6}>c_{0}$, and $c^{s t}(5,0)=$ $\frac{3}{20}<c_{0}$ (these computations were made in the proof of Proposition 4.2), we have

$$
K\left(c_{0}, 4,0\right), K\left(c_{0}, 6,0\right)>1 \text {, and } K\left(c_{0}, 5,0\right)=1 .
$$

Moreover, since $c^{s t}(n, a)$ is continuous in $a$ for a fixed $n$, for all positive and sufficiently small $a_{4}, a_{5}$, and $a_{6}$

$$
K\left(c_{0}, 4, a_{4}\right), K\left(c_{0}, 6, a_{6}\right)>1, \text { and } K\left(c_{0}, 5, a_{5}\right)=1
$$


Consequently:

Corollary 5.3: The stable number of countries is not monotone in the polarization degree. That is, while a simultaneous increase of both $n$ and $a$ reduces the polarization index $\gamma_{\alpha}(n, a)$, it does not necessarily decrease, or increase, the stable number $K(c, n, a)$ for a given $c$.

The example on which this corollary is based utilizes relatively high values of $c$. It turns out that for low values of $c$ the stable number does behave monotonically in the polarization index: it decreases with polarization, as we show in Proposition 5.4. Intuitively, this reflects the fact that in a very polarized society each cluster is relatively uniform, and hence, when separated from others, can exist as a separate and stable country even when the government cost is very low. Thus, for a wide range of low $c$, highly polarized $I$ should not be split into more countries than there are clusters, which keeps the stable number bounded. However, when the society is not polarized, and its members' preferences are spread uniformly, low $c$ necessitates a very fine partition to achieve stability, because of the wide spread of preferences.

Proposition 5.4: Given two integers $2 \leq n_{1} \leq n_{2}$ and $0 \leq a_{1} \leq a_{2} \leq \frac{1}{n_{2}}$, there exists $c\left(n_{1}, n_{2}, a_{1}, a_{2}\right)>0$ such that for every $0<c \leq c\left(n_{1}, n_{2}, a_{1}, a_{2}\right)$,

$$
K\left(c, n_{1}, a_{1}\right) \leq K\left(c, n_{2}, a_{2}\right)
$$

\section{$6 \quad$ Appendix}

We start with the following lemma:

Lemma A.1: If $k, n$ are integers with $1 \leq k \leq n-1$, then for the distribution $F_{n, 0}$

$$
D\left(\left[0, \frac{k}{n-1}\right]\right)=\frac{\left(\left[\frac{k}{2}\right]+1\right)\left(k-\left[\frac{k}{2}\right]\right)}{n(n-1)} .
$$




\section{Proof: Clearly}

$$
\begin{aligned}
D\left(\left[0, \frac{k}{n-1}\right]\right)= & D\left(\left[0, \frac{k}{n-1}\right], \frac{1}{2} \frac{k}{n-1}\right)=\frac{2}{n}\left(\frac{1}{2} \frac{k}{n-1}-0\right) \\
& +\frac{2}{n}\left(\frac{1}{2} \frac{k}{n-1}-\frac{1}{n-1}\right)+\ldots+\frac{2}{n}\left(\frac{1}{2} \frac{k}{n-1}-\frac{\left[\frac{k}{2}\right]}{n-1}\right) \\
= & \frac{2}{n} \frac{1}{2} \frac{k}{n-1}\left(\left[\frac{k}{2}\right]+1\right)-\frac{2}{n(n-1)}\left(1+2+\ldots+\left[\frac{k}{2}\right]\right) \\
= & \frac{\left(\left[\frac{k}{2}\right]+1\right)\left(k-\left\lfloor\frac{k}{2}\right\rfloor\right)}{n(n-1)} .
\end{aligned}
$$

Our second lemma provides a computational formula for the unity index of distributions in $\mathcal{F}$. Its proof relies on the result of Haimanko et al. (2004), stating that stability of the country is equivalent to its efficiency (the country is efficient if the total cost $^{6}$ incurred by its citizens is minimized when it is a united entity), and Proposition 3.3 of Haimanko et al. (2005), according to which the country is efficient if and only if splitting it into two independent regions does not decrease the total cost.

Lemma A.2: For every distribution $F_{n, a} \in \mathcal{F}$

$$
\begin{aligned}
c^{s t}(n, a) & =\max _{s \in[0,1]}[D(I)-D([0, s])-D([s, 1])] \\
& =D(I)-\min _{s \in[0,1]}[D([0, s])+D([s, 1])] .
\end{aligned}
$$

Proof of Proposition 3.1: Note that for $\alpha=0$, the index $\gamma_{0}$ defined by (1) for $F_{n, a}$ with $a>0$ and by (2) $F_{n, 0}$ is precisely the Gini inequality index. It is not a polarization index but it would be useful in our derivations. The index $\gamma_{0}(n, a)$ is simply the expected distance between two random points in $I$, each chosen according to $F_{n, a}$ and independently

\footnotetext{
${ }^{6}$ Obviously, this cost has two components: the aggregate transportation cost, and the government cost.
} 
of the other one. We claim that $\gamma_{0}(n, 0)=\frac{n+1}{3 n}$ for every $n$. Indeed, clearly

$$
\begin{aligned}
\gamma_{0}(n+1,0) & =\sum_{i=0}^{n} \sum_{j=0}^{n} \frac{1}{(n+1)^{2}}\left|\frac{i}{n}-\frac{j}{n}\right| \\
& =2 \sum_{i=0}^{n} \frac{i}{n(n+1)^{2}}+\sum_{i=0}^{n-1} \sum_{j=0}^{n-1} \frac{1}{(n+1)^{2}}\left|\frac{i}{n}-\frac{j}{n}\right| \\
& =\frac{1}{n+1}+\frac{n(n-1)}{(n+1)^{2}} \gamma_{0}(n, 0) .
\end{aligned}
$$

And

$$
\gamma_{0}(n, 0)=\frac{n+1}{3 n}
$$

obviously satisfies this recursive relation, with the initial condition $\gamma_{0}(2,0)=\frac{1}{2}$. It is also clear that

$$
\gamma_{0}\left(n, \frac{1}{n}\right)=\frac{1}{3}
$$

(recall that $F_{n, \frac{1}{n}}$ is the uniform distribution). Further, it follows from the definition of Gini index as the expected distance between two random points that $\gamma_{0}(n, \cdot)$ is an affine function of $a$ for fixed $n$, and therefore (4) and (5) imply that

$$
\gamma_{0}(n, a)=\frac{n+1-n a}{3 n}
$$

To shift from $\gamma_{0}(n, a)$ to $\gamma_{\alpha}(n, a)$ for positive values of $\alpha$, notice that

$$
\gamma_{\alpha}(n, a)=\left(\frac{1}{n a}\right)^{\alpha} \gamma_{0}(n, a)
$$

Thus,

$$
\gamma_{\alpha}(n, a)=\left(\frac{1}{n a}\right)^{\alpha} \frac{n+1-n a}{3 n}
$$

and

$$
\gamma_{\alpha}(n, 0)=\left(\frac{1}{n}\right)^{\alpha} \frac{n+1}{3 n} .
$$

Proof of Proposition 4.1: Note that the assertion of the proposition can be restated as follows: 
(i) if $n=4 m$ for $m \geq 1$, then

$$
c^{s t}(n, a)=\frac{m}{2(4 m-1)}(1-a)
$$

(ii) if $n=4 m+1$ for $m \geq 1$, then

$$
c^{s t}(n, a)=\frac{2 m+1}{4(4 m+1)}-\frac{a}{8} ;
$$

(iii) if $n=4 m+2$ for $m \geq 0$, then

$$
c^{s t}(n, a)=\frac{2 m^{2}+2 m+1}{2(4 m+1)(2 m+1)}-a \frac{2 m+3}{4(4 m+1)}
$$

(iv) if $n=4 m+3$ for $m \geq 0$, then

$$
c^{s t}(n, a)=\frac{m+1}{2(4 m+3)}-\frac{a}{8} .
$$

We first prove the equalities for the case of $a=0$. Start with (6), when $n=4 m$. Note that the minimum of $D([0, s])+D([s, 1])$ is attained at $s=\frac{1}{2}$ (or at any other point between $\frac{2 m-1}{4 m-1}$ and $\frac{2 m}{4 m-1}$ ). Indeed, if (say) $\frac{k-1}{4 m-1} \leq s<\frac{k}{4 m-1}<\frac{2 m-1}{4 m-1}$, then the following holds:

$$
\begin{aligned}
D([0, s])+D([s, 1]) & =D\left(\left[0, \frac{k-1}{4 m-1}\right]\right)+D\left(\left[\frac{k}{4 m-1}, 1\right]\right) \\
& =D\left(\left[0, \frac{k-1}{4 m-1}\right], \frac{k-1}{2(4 m-1)}\right)+D\left(\left[\frac{k}{4 m-1}, 1\right], \frac{1}{2}+\frac{k-1}{2(4 m-1)}\right) \\
& >D\left(\left[0, \frac{k}{4 m-1}\right], \frac{k-1}{2(4 m-1)}\right)+D\left(\left[\frac{k+1}{4 m-1}, 1\right], \frac{1}{2}+\frac{k-1}{2(4 m-1)}\right) \\
& \geq D\left(\left[0, \frac{k}{4 m-1}\right]\right)+D\left(\left[\frac{k+1}{4 m-1}, 1\right]\right) \\
& \geq \min _{s \in[0,1]}[D([0, s])+D([s, 1])] .
\end{aligned}
$$


Therefore

$$
\begin{aligned}
c^{s t}(4 m, 0) & =D(I)-\min _{s \in[0,1]}[D([0, s])+D([s, 1])] \\
& =D(I)-D\left(\left[0, \frac{1}{2}\right]\right)-D\left(\left[\frac{1}{2}, 1\right]\right) \\
& =D(I)-D\left(\left[0, \frac{2 m-1}{4 m-1}\right]\right)-D\left(\left[\frac{2 m}{4 m-1}, 1\right]\right) \\
& =D(I)-2 D\left(\left[0, \frac{2 m-1}{4 m-1}\right]\right)=(\text { using Lemma A.1) } \\
& =\frac{m}{4 m-1}-2 \frac{m}{4(4 m-1)}=\frac{m}{2(4 m-1)},
\end{aligned}
$$

which establishes (6) for $n=4 m$ and $a=0$.

Next, we consider the rest of the scenarios when $a=0$. Similarly to the previous case,

$$
\begin{aligned}
c^{s t}(4 m+1,0) & =D(I)-\min _{s \in[0,1]}[D([0, s])+D([s, 1])] \\
& =D(I)-D\left(\left[0, \frac{2 m-1}{4 m}\right]\right)-D\left(\left[\frac{2 m}{4 m}, 1\right]\right) \\
& =D(I)-D\left(\left[0, \frac{2 m-1}{4 m}\right]\right)-D\left(\left[0, \frac{2 m}{4 m}\right]\right) \\
& =\frac{(2 m+1) 2 m}{(4 m+1) 4 m}-\frac{m^{2}}{(4 m+1) 4 m}-\frac{(m+1) m}{(4 m+1) 4 m} \\
& =\frac{2 m+1}{4(4 m+1)},
\end{aligned}
$$

and (7) is also established. Further,

$$
\begin{aligned}
c^{s t}(4 m+2,0) & =D(I)-\min _{s \in[0,1]}[D([0, s])+D([s, 1])] \\
& =D(I)-D\left(\left[0, \frac{2 m}{4 m+1}\right]\right)-D\left(\left[\frac{2 m+1}{4 m+1}, 1\right]\right) \\
& =D(I)-2 D\left(\left[0, \frac{2 m}{4 m+1}\right]\right) \\
& =\frac{(2 m+1)^{2}}{(4 m+2)(4 m+1)}-2 \frac{m(m+1)}{(4 m+2)(4 m+1)} \\
& =\frac{2 m^{2}+2 m+1}{2(4 m+1)(2 m+1)},
\end{aligned}
$$


which shows (8). And finally,

$$
\begin{aligned}
c^{s t}(4 m+3,0) & =D(I)-\min _{s \in[0,1]}[D([0, s])+D([s, 1])] \\
& =D(I)-D\left(\left[0, \frac{2 m}{4 m+2}\right]\right)-D\left(\left[\frac{2 m+1}{4 m+2}, 1\right]\right) \\
& =D(I)-D\left(\left[0, \frac{2 m}{4 m+2}\right]\right)-D\left(\left[0, \frac{2 m+1}{4 m+2}\right]\right) \\
& =\frac{(2 m+1)(2 m+2)}{(4 m+3)(4 m+2)}-\frac{m(m+1)}{(4 m+3)(4 m+2)}-\frac{(m+1)^{2}}{(4 m+3)(4 m+2)} \\
& =\frac{m+1}{2(4 m+3)},
\end{aligned}
$$

and hence (9) is established as well.

It remains to prove the four equalities for $\left\{F_{n, a}\right\}_{n \geq 2, a \in\left(0, \frac{1}{n}\right]}$. Note that each such distribution is symmetric around $\frac{1}{2}$ and satisfies GEM (the "gradually escalating median" condition, set forth in Le Breton and Weber (2003). This condition requires that there be a (nondecreasing) selection of a median, $l(t)$, in every subinterval $[0, t]$, such that $l^{\prime}(t) \leq 1$ for almost every $t$. And it obviously holds for every $F_{n, a}$ for $n \geq 2, a \in\left(0, \frac{1}{n}\right]$, since one can consider

$$
l(t)=\left\{\begin{array}{cc}
\frac{t}{2}, & \text { if } t \in\left[k \frac{1-a}{n-1}, k \frac{1-a}{n-1}+a\right] \text { and } k \text { is even; } \\
\frac{a}{2}-\frac{1}{2} \frac{1-a}{n-1}+\frac{t}{2}, & \text { if } t \in\left[k \frac{1-a}{n-1}, k \frac{1-a}{n-1}+a\right] \text { and } k \text { is odd; } \\
\frac{k}{2} \frac{1-a}{n-1}+\frac{a}{2}, & \text { if } t \in\left[k \frac{1-a}{n-1}+a,(k+1) \frac{1-a}{n-1}\right] \text { and } k \text { is even; } \\
t-\frac{k+1}{2} \frac{1-a}{n-1}, & \text { if } t \in\left[k \frac{1-a}{n-1}+a,(k+1) \frac{1-a}{n-1}\right] \text { and } k \text { is odd }
\end{array}\right.
$$

According to Proposition 4.1 of Haimanko et al. (2005),

$$
c^{s t}(n, a)=\frac{1}{2}-4 \int_{l\left(\frac{1}{2}\right)}^{\frac{1}{2}} t f_{n, a}(t) d t .
$$

Due to the particular form of $f_{n, a}(t)$ and $l\left(\frac{1}{2}\right)$, this implies that $c^{s t}(n, a)=q(n) a+r(n)+s(n) \frac{1}{a}$. However, since $0 \leq c^{s t}(n, a) \leq 1$ for all $a$, it follows that $c^{s t}(n, a)$ has the form

$$
c^{s t}(n, a)=q(n) a+r(n),
$$

i.e., it is an affine function of $a$ for fixed $n$. Equality (10) also holds when $a=0$, since the expression $\min _{s \in[0,1]}[D([0, s])+D([s, 1])]$ is continuous in the distribution $F$, as was established in Lemma A.7 in Haimanko et al. (2004). 
Since $F_{n, \frac{1}{n}}$ is uniform on $[0,1]$,

$$
q(n) \frac{1}{n}+r(n)=c^{s t}\left(n, \frac{1}{n}\right)=D(I)-2 D\left(\left[0, \frac{1}{2}\right]\right)=\int_{0}^{1}\left|t-\frac{1}{2}\right| d t-2 \int_{0}^{\frac{1}{2}}\left|t-\frac{1}{4}\right| d t=\frac{1}{8} .
$$

We also know that

$$
r(n)=q(n) \cdot 0+r(n)=c^{s t}(n, 0)
$$

and therefore

$$
c^{s t}(n, a)=n\left(\frac{1}{8}-c^{s t}(n, 0)\right) a+c^{s t}(n, 0) .
$$

Substituting the values of $c^{s t}(n, 0)$ that have been computed above into the above equality yields (6), (7), (8), and (9).

Proof of Proposition 4.2: (i). Follows immediately from Proposition 4.1 and Corollary 3.2 .

(ii) and (iii). Consider the case where $a=0$. When $n$ increases, the distribution $F_{n, 0}$ becomes less polarized and, in the limit, converges to the uniform distribution. The unity index $c^{s t}(n, 0)$ clearly converges to $\frac{1}{8}$ as $n \rightarrow \infty$. By Proposition 4.1 , it decreases for low values of $n: c^{s t}(2,0)=\frac{1}{2}, c^{s t}(3,0)=\frac{1}{6}, c^{s t}(4,0)=\frac{1}{6}, c^{s t}(5,0)=\frac{3}{20}$. However, $c^{s t}(6,0)=\frac{1}{6}>c^{s t}(5,0)$, and thus a spike in the unity index is observed on its way down to $\frac{1}{8}$, despite the decreasing polarization and increasing uniformity of the distribution. This spike is recurrent: clearly,

$$
c^{s t}(4 m+1,0), c^{s t}(4 m+3,0)<c^{s t}(4 m+2,0),
$$

and even

$$
c^{s t}(4 m-1,0), c^{s t}(4 m, 0) \leq c^{s t}(4 m+2,0)
$$

(equality occurs only for $m=1$ ). Moreover, if $a_{1}, a_{2}$, and $a_{3}$ are positive and sufficiently small, the inequality

$$
c^{s t}\left(4 m+1, a_{1}\right), c^{s t}\left(4 m+3, a_{2}\right)<c^{s t}\left(4 m+2, a_{3}\right)
$$


holds as well, due to continuity of $c^{s t}(n, a)$ for a fixed $n$. This establishes (ii) and (iii) of the proposition.

Proof of Proposition 4.3: Fix $a>0$. Note that $c^{s t}(n+1, a) \leq c^{s t}(n, a)$ for all feasible $n$, except possibly for those that have the form $n=4 m+1$. Consider the expression $c^{s t}(4 m+2, a)-c^{s t}(4 m+1, a)$. By Proposition 4.1, this difference is equal to

$$
\frac{1}{8(4 m+1)}\left(\frac{2}{2 m+1}-5 a\right)
$$

Thus, $c^{s t}(n+1, a)-c^{s t}(n, a) \leq 0$ for $n>n(a)$, where $n(a)=\frac{4}{5 a}$.

Proof of Proposition 5.4: This follows immediately from Proposition 3.1 of Haimanko et al. (2004). Indeed, according to this proposition,

$$
\lim _{c \rightarrow 0} K(c, n, a) \sqrt{c}=\frac{1}{2} \int_{0}^{1} \sqrt{f_{n, a}(t)} d t=\frac{1}{2} \sqrt{n a}
$$

if $a>0$, and clearly

$$
\lim _{c \rightarrow 0} K(c, n, 0)=n
$$

if $a=0$.

\section{References}

Alesina, A. and E. Spolaore (1997) "On the Number and Size of Nations", Quarterly Journal of Economics 113, 1027-1056.

Alesina, A., R. Baqir and W. Easterly (1999) "Public Goods and Ethnic Divisions", Quarterly Journal of Economics, 114, 1243-1284.

Alesina, A., R. Baqir and C. Hoxby (2004) "Political Jurisdictions in Heterogeneous Communities", Journal of Political Economy, 112, 348-396.

Caselli, F. and W.B. Coleman (2002) "On the Theory of Ethnic Conflict", mimeo. 
Duclos, J. Y., Esteban, J. and D. Ray (2004) "Polarization: Concepts, Measurement, Estimation," Econometrica 72, 1737-1772.

Esteban, J. and D. Ray (1994) "On the Measurement of Polarization," Econometrica 62, 819-852.

Esteban, J. and D. Ray (1999) "Conflict and Distribution," Journal of Economic Theory $87(2), 379-415$.

Gershenson, D. and H.I. Grossman (1999) "Civil Conflict: Ended or Never Ending?", Journal of Conflict Resolution 44, 807-921.

Grossman, H.I. (1991) "A General equilibrium Model of Insurrections", American Economic Review 81, 912-921.

Haimanko, O., M. Le Breton and S. Weber (2005) "Transfers in a Polarized Country: Bridging the Gap Between Efficiency and Stability," Journal of Public Economics 89, 12771303.

Haimanko, O., M. Le Breton and S. Weber (2004) "Voluntary Formation of Communities for the Provision of Public Projects," Journal of Economic Theory 115, 1-34.

Le Breton, M. and S. Weber (2003) "The Art of Making Everybody Happy: How to Prevent a Secession", IMF Staff Papers 50 (3), 403-435.

Wang, Y.Q. and K.Y. Tsui (2000), "Polarization Orderings and New Classes of Polarization Indices," Journal of Public Economic Theory 2, 349-363. 